\title{
Applying exclusion likelihoods from LHC searches to extended Higgs sectors
}

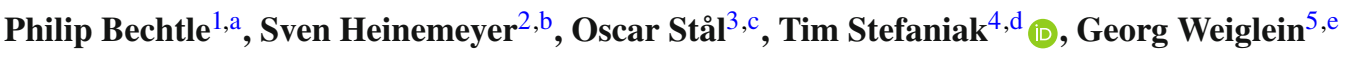 \\ ${ }^{1}$ Physikalisches Institut der Universität Bonn, Nußallee 12, 53115 Bonn, Germany \\ ${ }^{2}$ Instituto de Física de Cantabria (CSIC-UC), Santander, Spain \\ ${ }^{3}$ Department of Physics, The Oskar Klein Centre, Stockholm University, 10691 Stockholm, Sweden \\ ${ }^{4}$ Santa Cruz Institute for Particle Physics (SCIPP), University of California, Santa Cruz, CA 95064, USA \\ ${ }^{5}$ Deutsches Elektronen-Synchrotron DESY, Notkestraße 85, 22607 Hamburg, Germany
}

Received: 4 August 2015 / Accepted: 28 August 2015 / Published online: 15 September 2015

(C) The Author(s) 2015. This article is published with open access at Springerlink.com

\begin{abstract}
LHC searches for non-standard Higgs bosons decaying into tau lepton pairs constitute a sensitive experimental probe for physics beyond the Standard Model (BSM), such as supersymmetry (SUSY). Recently, the limits obtained from these searches have been presented by the CMS collaboration in a nearly model-independent fashion - as a narrow resonance model - based on the full $8 \mathrm{TeV}$ dataset. In addition to publishing a $95 \%$ C.L. exclusion limit, the full likelihood information for the narrow resonance model has been released. This provides valuable information that can be incorporated into global BSM fits. We present a simple algorithm that maps an arbitrary model with multiple neutral Higgs bosons onto the narrow resonance model and derives the corresponding value for the exclusion likelihood from the CMS search. This procedure has been implemented into the public computer code HiggsBounds (version 4.2 .0 and higher). We validate our implementation by cross-checking against the official CMS exclusion contours in three Higgs benchmark scenarios in the Minimal Supersymmetric Standard Model (MSSM), and find very good agreement. Going beyond validation, we discuss the combined constraints of the $\tau \tau$ search and the rate measurements of the SM-like Higgs at $125 \mathrm{GeV}$ in a recently proposed MSSM benchmark scenario, where the lightest Higgs boson obtains SM-like couplings independently of the decoupling of the heavier Higgs states. Technical details for how to access the likelihood information within HiggsBounds
\end{abstract}

\footnotetext{
a e-mail: bechtle@physik.uni-bonn.de

b e-mail: Sven.Heinemeyer@cern.ch

c e-mail: oscar.stal@fysik.su.se

de-mail: tistefan@ucsc.edu

e e-mail: Georg.Weiglein@ desy.de
}

are given in the appendix. The program is available at http:// higgsbounds.hepforge.org.

\section{Introduction}

The search for Higgs bosons [1-6] continues to be a cornerstone of the physics program at the Large Hadron Collider (LHC). After the discovery of a Higgs boson by ATLAS [7] and CMS [8] it is crucial to find out whether the detected particle is part of a Higgs sector that contains several physical states. Higgs sectors of this kind are predicted in many theories of physics beyond the Standard Model (SM). For the understanding of the mechanism of electroweak symmetry breaking two complementary experimental endeavors are important: On the one hand the precise determination of the properties of the Higgs signal detected at around $125 \mathrm{GeV}$, and on the other hand the search for additional Higgs bosons. Both are crucial in the quest to identify the underlying physics. The existing limits from the Higgs searches at LEP, the Tevatron and the LHC already put very important constraints on the parameter spaces of different models that provide a Higgs-like state compatible with the detected signal. More data on both the detected signal and on searches for additional Higgs bosons will further enhance the sensitivity for discriminating possible scenarios of new physics from the SM and from each other.

In order to facilitate the available experimental information from the Higgs searches at LEP, the Tevatron and the LHC, expressed in terms of relatively model-independent cross-section limits for testing a wide variety of theoretical models, the program HiggsBounds [9-12] has been developed. Experimental information on the Higgs signal detected at a mass value of around $125 \mathrm{GeV}$ is utilized in the sister program HiggsSignals [13] for testing the theoret- 
ical predictions from any kind of Higgs sector. The experimental information on the detected signal incorporated in HiggsSignals is turned into a $\chi^{2}$ likelihood, which is suitable for the inclusion into global fits (see, e.g., Refs. [1419]), where in addition many other observables are taken into account. In contrast, exclusion limits have traditionally been presented in terms of $95 \%$ C.L. limits, which a priori only provide the information whether a particular parameter point is excluded or not at the $95 \%$ C.L. by the considered search channel. In a global fit, where the predictions of a model are confronted with a large number of observables, it would usually be too restrictive to disregard a certain parameter point just because it falls outside of the $95 \%$ C.L. region of a single search channel. In fact, testing a large variety of observables one would expect that the measured values of some observables lie outside of the respective $95 \%$ C.L. regions for purely statistical reasons. It would therefore be very desirable if also negative experimental outcomes from Higgs searches were provided in terms of the likelihood information in the relevant parameters, instead of a simple binary rejection or acceptance at a certain confidence level (C.L.). Up to now, likelihood information was available in HiggsBounds only for the results from the LEP Higgs searches [12], while for all search channels at the Tevatron and LHC only $95 \%$ C.L. limits have been accessible. We report here on significant progress in this direction for the LHC Higgs boson search in the $\tau^{+} \tau^{-}$final state, which plays a central role in the search for additional Higgs bosons.

Many models that can accommodate a SM-like Higgs boson at $125 \mathrm{GeV}$, such as the Minimal Supersymmetric Standard Model (MSSM) or the various types of Two-HiggsDoublet Models (2HDM), predict additional Higgs bosons that decay predominantly into SM fermions. Therefore, LHC searches for new neutral Higgs bosons decaying to $\tau^{+} \tau^{-}$play a crucial role. In particular within the MSSM, these searches lead to large excluded regions in the parameter space. The highest experimental sensitivity occurs for smaller values of the $\mathcal{C P}$-odd Higgs boson mass, $M_{A}$, and larger values of $\tan \beta$, the ratio of the two vacuum expectation values [20,21].

One complication that arises for this search channel is the fact that two different production modes, gluon fusion and $b$ quark associated production, can both be important. Their individual contributions to the signal rate can vary strongly over the parameter space. Since the acceptances of these two channels can also be very different, a two-dimensional cross section interpretation for the $\tau^{+} \tau^{-}$final state is desirable as a basis for (close to) model-independent exclusion limits. Recently, the CMS collaboration has published the likelihood information for their Higgs boson search in the $\tau^{+} \tau^{-}$final state [20]. The likelihood is given as a function of the two relevant Higgs production channels, gluon fusion and $b$ quark associated production, for various mass values of the narrow resonance assumed for the signal model.
In this paper we investigate the application of this new experimental information for testing the theoretical predictions of extended Higgs sectors and its incorporation in global fits. We develop a simple algorithm that maps an arbitrary model with in general several neutral Higgs bosons onto the narrow resonance model. In this way the corresponding value of the exclusion likelihood from the CMS search for the tested model can be determined. We furthermore describe the inclusion of this likelihood information into the publicly available Fortran code HiggsBounds [9-12]. For nearly any model under consideration, HiggsBounds provides an evaluation of the exclusion likelihood for a model parameter point based on the information from [20]. While the new likelihood information goes well beyond the standard test whether a particular parameter point is excluded at the $95 \%$ C.L., the likelihood information can also be employed to run HiggsBounds in this "standard" mode. In this case, HiggsBounds determines the parameter region that is excluded at the $95 \%$ C.L. based on all available searches, including the new $\tau^{+} \tau^{-}$result from CMS. The new version of HiggsBounds can be used together with its sister program HiggsSignals [13] in order to take into account both the information from search limits and from the detected signal for a comprehensive test of Higgs phenomenology. Both codes are available at: http://higgsbounds.hepforge.org.

The paper is organized as follows. In Sect. 2 we briefly summarize the experimental results that are used as input for our investigations. Details of the employed algorithm and the implementation of the exclusion likelihood of Ref. [20] into HiggsBounds are given in Sect. 3. The validation in various MSSM Higgs benchmark scenarios is discussed in Sect. 4. As an application, in Sect. 5 we investigate the constraints on a certain benchmark scenario in the MSSM that are obtained from using the new exclusion likelihood in combination with the information on the detected signal incorporated in HiggsSignals. We conclude in Sect. 6. Finally, all relevant information needed to run HiggsBounds to obtain the likelihood information for the $\tau^{+} \tau^{-}$Higgs search channel for any parameter point under investigation are contained in an Appendix.

\section{Experimental results}

This section briefly summarizes the experimental results from the CMS non-standard Higgs search in the $\tau \tau$ final state [20], that we have used as starting point for our investigation and that we have implemented in HiggsBounds. The search analysis of CMS is carried out in two separate selection categories: One requiring the presence of at least one $b$-tagged jet, and one without the presence of a $b$-tag. The former category is enriched by the production of a Higgs boson, denoted generically by $\phi$, in association with two $b$ 
quarks, $g g \rightarrow b \bar{b} \phi$, while the latter is dominated by the gluon fusion process, $g g \rightarrow \phi$. Hence, the search features sensitivity to the two different production modes separately, which enables the presentation of the search results in terms of individual signal strengths in both production modes for all tested Higgs boson masses. Separate information on the two production modes is an indispensable ingredient for enabling the presentation of (close to) model-independent exclusion limits or measurements, in case of a discovery.

The data is further classified in categories defined by the two $\tau$ lepton decay modes: $e \tau_{h}, \mu \tau_{h}, e \mu, \mu \mu$ and $\tau_{h} \tau_{h}$, where $\tau_{h}$ denotes a hadronically decaying $\tau$ lepton. Using a maximum likelihood technique, an estimator for the true $\tau \tau$ invariant mass, $m_{\tau \tau}$, is reconstructed from the momenta of the visible $\tau$ decay products and the missing transverse energy in the event. The uncertainty of the $m_{\tau \tau}$ reconstruction is estimated to be around $20 \%$ when averaged over all decay modes [20].

The resulting $m_{\tau \tau}$ spectrum in all categories ( $b$-tag and $\tau$ decay) separately is then subject to a profile likelihood analysis [22], where the background parametrization, obtained from control region data and Monte Carlo simulation, and the signal shape parametrization are fitted simultaneously to the reconstructed mass spectrum. The fit is performed individually for test masses $m_{\phi}$ between $90 \mathrm{GeV}$ and $1 \mathrm{TeV}$, and the results are interpolated between the test masses.

CMS interprets the results both in a nearly modelindependent way ${ }^{1}$ for a single narrow resonance $\phi$, and, in a model-specific context, in the MSSM, where three neutral Higgs bosons $h, H$ and $A$ potentially comprise the signal. The latter interpretation performs a likelihood ratio hypothesis test for the two hypotheses of a single SM-like Higgs boson at $m_{h}=125 \mathrm{GeV}$ with exact SM properties and, alternatively, for the signal consisting of all three neutral Higgs bosons of the MSSM. In the latter case, the $m_{\tau \tau}$ distributions of the $h / H / A \rightarrow \tau \tau$ decays are combined before the calculation of the likelihood. Note that, whereas the modelspecific limits for the MSSM are based on the full integrated luminosity of the combined $7+8 \mathrm{TeV}$ dataset, the results for the single narrow resonance model are obtained from only the $8 \mathrm{TeV}$ dataset.

Since HiggsBounds is designed to test any extended Higgs sector, with any coupling properties of the $125 \mathrm{GeV}$ Higgs candidate (if the model under consideration provides such a candidate; obviously the phenomenological interest in other models is rather limited) and any masses and properties of the remaining Higgs spectrum, the nearly modelindependent single resonance results are chosen for the

\footnotetext{
1 The presentation of the search results in terms of a limit on the inclusive total cross section times branching ratio inevitably involves a slight model dependence from the extrapolation to the inclusive quantity. In other words, the expectation of the kinematic distributions of the signal and/or background is model dependent.
}

implementation in HiggsBounds. On the one hand, this is the only possibility unless one is willing to adopt further model-dependent assumptions. On the other hand, it will in general yield weaker, i.e. more conservative, limits than a dedicated analysis taking into account the full structure of the considered model. For example, in the MSSM this will yield a conservative limit whenever either $m_{H / A} \approx m_{h}$, or, more generally, whenever the model predicts that more than one Higgs boson have a non-negligible signal yield and contribute in different regions in $m_{\tau \tau}$. Since the likelihood is constructed for single resonances, such a case cannot be properly reconstructed from the likelihood. However, if e.g. the heavy MSSM Higgs bosons $H$ and $A$ contribute at the same point in $m_{\tau \tau}$, their signal rates can be added before interpreting the likelihood. In this case the implementation is not necessarily conservative. A detailed study on the applicability of these limits to the MSSM benchmarks is presented in Sect. 4.

The profile likelihood analysis follows the standard implementation: The test statistics is given by

$q_{\mu}=-2 \ln \frac{\mathcal{L}\left(N \mid \mu \cdot s(m)+b, \hat{\theta}_{\mu}\right)}{\mathcal{L}(N \mid \hat{\mu} \cdot s(m)+b, \hat{\theta})}$.

Here $N$ is the number of measured events, $b$ and $s(m)$ the number of expected background and signal events for a given resonance mass hypothesis $m, \mu$ the signal strength modifier, and $\theta$ are the nuisance parameters describing the systematic uncertainties. $\hat{\theta}_{\mu}$ maximizes the likelihood in the numerator given a certain value of $\mu$, whereas the likelihood reaches its global maximum at $\hat{\mu}$ and $\hat{\theta}$, which is given in the denominator. The constraint $0 \leq \hat{\mu} \leq \mu$ is employed to not penalize the model for a possible excess of the data over the signal plus background prediction. It should be noted that the signal yield contains two independent components, corresponding to the two production modes $g g \rightarrow \phi$ and $g g \rightarrow b \bar{b} \phi$. Thus, $\mu$ and $s(m)$ are two-component vectors.

Using toy Monte Carlo techniques or asymptotic expressions for large statistics, the expected probability distributions $P\left(q_{\mu} \mid\right.$ hypothesis $)$ can be constructed for the test statistics given above. In the model-independent analysis used here, the hypothesis either consists of $H_{1}=\mu \cdot s(m)+b$ for the case of the presence of a single narrow resonance with a given mass $m$ and signal yield $\mu \cdot s(m)$, and of $H_{2}=b$ for the background. Using these hypothesis definitions and the observed value of the likelihood ratio, $q_{\mu}^{\text {obs }}$, given by Eq. (1) with $N$ given by the actual observed number of events, $N=N_{\text {obs }}$, the likelihood ratio technique can be used to define the $\mathrm{CL}_{s}$ as

$\mathrm{CL}_{s}(\mu)=\frac{P\left(q_{\mu} \geq q_{\mu}^{\mathrm{obs}} \mid \mu \cdot s(m)+b\right)}{P\left(q_{\mu} \geq q_{\mu}^{\mathrm{obs}} \mid b\right)}$.

In a stand-alone search, the criterion $\mathrm{CL}_{s} \leq \alpha$ is then used to exclude the presence of a signal at $1-\alpha$ confi- 
dence level (C.L.). For the expected limit, the observed data in the calculation of $q_{\mu}^{\mathrm{obs}}$ is replaced by the median of the background-only expectation for $q_{\mu}$. A model-independent limit, e.g. at the $95 \%$ C.L., can then be derived by varying $\mu$ until $\mathrm{CL}_{s}=0.05$. The value $\mu$ at which this happens then represents the signal strength modifier which is just allowed at the $95 \%$ C.L.

For any given model, HiggsBounds reconstructs the predicted signal yield $s(m)$ from the theoretical input provided by the user, and obtains the corresponding value of the test statistics $q_{\mu=1}$ (or simply denoted $q_{\text {model }}$ ) from the CMS likelihood data. The details of this procedure will be described in the following section. Note that HiggsBounds directly employs the expectation and observation of the test statistics, $q_{\mu}^{\exp }$ and $q_{\mu}^{\text {obs }}$, respectively, as the provided CMS data does not allow for a full reconstruction of the $\mathrm{CL}_{s}$ value. Nevertheless, in the limit of large numbers, the test statistics can be approximated by a chi-squared differences function above minimum, $q_{\mu} \approx \Delta \chi^{2}=\chi^{2}-\chi_{\min }^{2}$, as

$\chi^{2} \approx-2 \ln \mathcal{L}\left(N \mid \mu \cdot s(m)+b, \hat{\theta}_{\mu}\right)$,
$\chi_{\min }^{2} \approx-2 \ln \mathcal{L}(N \mid \hat{\mu} \cdot s(m)+b, \hat{\theta})$.

Thus, for example, we approximately obtain the twodimensional limit at the 68 and $95 \%$ C.L. when the test statistics $q_{\mu}$ takes the values 2.28 and 5.99, respectively.

As an example, we show the observed likelihood distribution, $q_{\mu}^{\text {obs }}$, for test masses of 125 and $300 \mathrm{GeV}$ in Fig. 1. We also indicate the approximated 68 and $95 \%$ C.L. limits by contour lines. It should be kept in mind that these contours are only for illustrational purposes. In HiggsBounds the full likelihood information $q_{\mu}$ is used, and the specific limit at a certain C.L. can be easily obtained from this information.

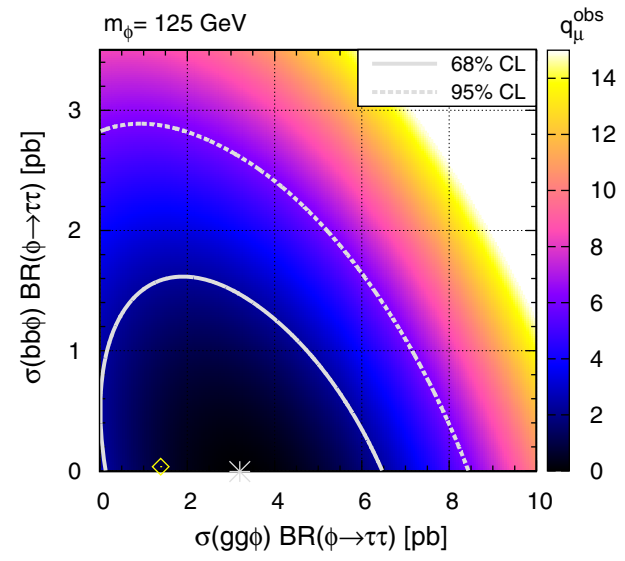

(a)

Fig. 1 Results for the observed exclusion likelihood, $q_{\mu}^{\text {obs }}$, from the CMS $\phi \rightarrow \tau \tau$ analysis [20], assuming a narrow resonance mass, $m_{\phi}$, of $125 \mathrm{GeV}$ (a) and $300 \mathrm{GeV}(\mathbf{b})$. The solid (dashed) lines are obtained at $q_{\mu}^{\text {obs }}=2.28(5.99)$ and indicate the approximate $68 \%$ (95\%) C.L.
The implementation of the likelihood for the CMS $\phi \rightarrow$ $\tau \tau$ search differs in two significant ways from the implementation of the LEP Higgs search $\chi^{2}$ in HiggsBounds, which is already available since version 2.0.0 [10]. In the LEP implementation, each Higgs search channel, comprised of one production mode and one Higgs boson decay mode, is treated separately, thus no combination of production modes is applied or possible for the user. In addition, in the LEP implementation the $\chi^{2}$ is estimated from the $\mathrm{CL}_{s+b}$ value in each channel at the given signal strength prediction using Gaussian approximations. In contrast, for the CMS $\phi \rightarrow \tau \tau$ search the exact values of the test statistics $q_{\mu}$ as presented by CMS are used and properly combined for both production modes.

\section{Likelihood reconstruction for extended Higgs sectors}

For the construction of the exclusion likelihood from the $H \rightarrow \tau \tau$ search, we make use of the following quantities: For each neutral Higgs boson, $h_{i}(i=1, \ldots, N)$, in a model with $N$ neutral Higgs bosons, we have a prediction of the mass, $m_{i}$ (where the relevant range is currently $\left.m_{i} \in[90,1000] \mathrm{GeV}\right)$, the gluon fusion production cross section, $\sigma\left(g g \rightarrow h_{i}\right)$, the cross section for production in association with $b$ quarks, $\sigma\left(g g \rightarrow b \bar{b} h_{i}\right)$, and the branching fraction $\mathrm{BR}\left(h_{i} \rightarrow \tau \tau\right)$.

The main algorithm for the likelihood reconstruction proceeds as follows:

1. Signal rates of multiple Higgs bosons that cannot be resolved by the experimental analysis are added. We thus combine the signal predictions for two Higgs boson $h_{i}$ and $h_{j}(j \neq i)$, if

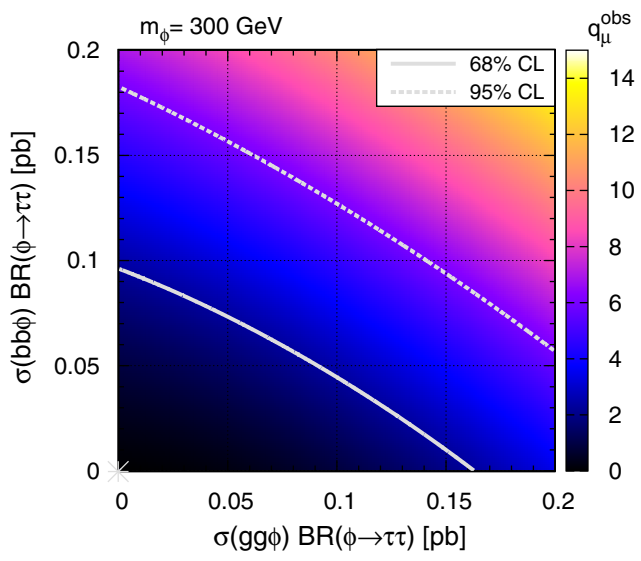

(b)

allowed regions of a Higgs boson signal. The gray asterisk indicates the location of the global maximum of the likelihood. In (a) the yellow hollow diamond indicates the prediction of a Higgs boson at $125 \mathrm{GeV}$ with SM signal strength 
$\left|m_{i}-m_{j}\right| \leq 20 \% \cdot \max \left(m_{i}, m_{j}\right)$

Each Higgs boson can appear in different such combinations. For each combination, also called Higgs cluster and labeled with capital characters in the following, we evaluate the physical quantities as follows: We assume that the total rates are given by the incoherent sum of the signal rates of the individual Higgs bosons in the cluster,

$$
\begin{aligned}
& \sigma\left(g g \rightarrow h_{I} \rightarrow \tau \tau\right) \\
& \quad=\sum_{k} \sigma\left(g g \rightarrow h_{k}\right) \cdot \mathrm{BR}\left(h_{k} \rightarrow \tau \tau\right), \\
& \sigma\left(g g \rightarrow b \bar{b} h_{I} \rightarrow \tau \tau\right) \\
& \quad=\sum_{k} \sigma\left(g g \rightarrow b \bar{b} h_{k}\right) \cdot \mathrm{BR}\left(h_{k} \rightarrow \tau \tau\right) .
\end{aligned}
$$

The cluster mass, $m_{I}$, is determined by a signal strengths weighted mass average

$m_{I}=\frac{\sum_{k}\left[\sigma\left(g g \rightarrow h_{k}\right)+\sigma\left(g g \rightarrow b \bar{b} h_{k}\right)\right] \cdot \mathrm{BR}\left(h_{k} \rightarrow \tau \tau\right) \cdot m_{k}}{\sum_{k}\left[\sigma\left(g g \rightarrow h_{k}\right)+\sigma\left(g g \rightarrow b \bar{b} h_{k}\right)\right] \cdot \mathrm{BR}\left(h_{k} \rightarrow \tau \tau\right)}$.

The sums in Eqs. (6)-(8) run over all Higgs bosons $h_{k}$ combined in the cluster. In case there is no $h_{j}$ that fulfills Eq. (5) for a given $h_{i}$, the cluster is formed solely by the Higgs boson $h_{i}$. It should be noted that taking the incoherent sum of the contributions of the different Higgs bosons involves an approximation. While it is exact in the case of two different $\mathcal{C P}$ eigenstates, e.g. $A$ and $H$ in the MSSM, in general interference contributions can be important $[23,24]$. An extension of HiggsBounds that enables the implementation of interference effects of nearby resonances in a generalized narrow-width approximation is currently under development, see also Ref. [25].

2. In the second step, the expected and observed likelihood values, $q_{\text {model }}^{\exp }$ and $q_{\text {model }}^{\text {obs }}$, respectively, for each Higgs cluster $h_{I}$ are evaluated from the experimental likelihood data grid. The likelihood is first evaluated for the rate values $\sigma\left(g g \rightarrow h_{I} \rightarrow \tau \tau\right)$ and $\sigma\left(g g \rightarrow b \bar{b} h_{I} \rightarrow \tau \tau\right)$, obtained through Eqs. (6), (7), in the mass-neighboring data slices, i.e. at the nearest grid mass values below and above $m_{I}$, denoted by $m_{-}$and $m_{+}$, respectively. The likelihood value at the predicted cluster mass $m_{I}$ is then obtained through linear interpolation:

$q\left(h_{I}\right)=\frac{q_{-} \cdot\left(m_{+}-m_{I}\right)+q_{+} \cdot\left(m_{I}-m_{-}\right)}{m_{+}-m_{-}}$.

Here $q_{+/-}$denote the values of the test statistics obtained at the neighboring grid above or below the predicted mass
$m_{I}$ [we omitted the subscript 'model' for simplicity in Eq. (9)]. These are obtained, in each case, through bilinear interpolation within the two-dimensional likelihood planes of the provided CMS data.

3. The steps 1 and 2 are repeated until all $N$ neutral Higgs bosons have been evaluated as part of at least one Higgs cluster.

4. Once all likelihoods have been evaluated, the most sensitive analysis application is determined from the resulting expected likelihood, $q_{\text {model }}^{\exp }$, i.e. the cluster $h_{I}^{\max }$ is selected for which $q_{\text {model }}^{\exp }\left(h_{I}\right)$ is maximal. The observed exclusion likelihood, $q_{\text {model }}^{\text {obs }}$, is then used only for this cluster, and provides the final result.

Following this algorithm, the full likelihood from the CMS $\phi \rightarrow \tau \tau$ analysis for both the expected and observed exclusion can be directly obtained within HiggsBounds for any tested model. This is carried out via Fortran subroutines. For a technical documentation see Appendix.

The use of this likelihood information is complementary to the other type of information contained in a full HiggsBounds application, which considers exclusion limits from many other Higgs searches from the LEP, Tevatron and LHC experiments. As an alternative to using the full likelihood, we therefore also provide the option to reconstruct a limit at $95 \%$ C.L. and use this in the "standard" HiggsBounds operation. For clarity, we now repeat some elements of how this works [12]: In the statistical procedure, HiggsBounds first determines the most sensitive analysis to the model by picking the analysis application, for which the ratio between the model-predicted signal rate, $S_{\text {predicted, over the expected }}$ upper limit on the signal rate, $S_{\text {expected }}^{95 \% \mathrm{CL}}$,

$r_{\text {expected }} \equiv \frac{S_{\text {predicted }}}{S_{\text {expected }}^{95 \% \mathrm{CL}}}$

is maximized. After the most sensitive analysis has been determined, the model prediction is confronted with the observed exclusion limit of this particular analysis, $S_{\text {observed }}^{95 \% \mathrm{CL}}$. The model is considered to be excluded at the $95 \%$ C.L., if

$r_{\text {observed }} \equiv \frac{S_{\text {predicted }}}{S_{\text {observed }}^{95 \% \text { CL }}}>1$

For the CMS $\phi \rightarrow \tau \tau$ analysis described above, $S_{\text {expected }}^{95 \% \mathrm{CL}}$ and $S_{\text {observed }}^{95 \% \mathrm{CL}}$ are a priori not known and need to be determined from the implemented likelihood distribution. In a numerical procedure, we therefore scale the model-predicted $g g \rightarrow \phi \rightarrow \tau \tau$ and $g g \rightarrow b \bar{b} \phi \rightarrow \tau \tau$ rates with a universal factor $\mu$ until the obtained expected/observed likelihood $q_{\mu}^{\text {exp/obs }}$ values are equal to 5.99, corresponding to the two- 
dimensional $95 \%$ C.L. interval. ${ }^{2}$ The so-obtained scale factors, $\mu_{95 \% \mathrm{CL}}^{\text {exp/obs }}$, are then identified with the expected/observed $95 \%$ C.L. upper limits on the signal rate, respectively, which enter Eqs. (10) and (11). In this way, the likelihood-based results from the CMS $\phi \rightarrow \tau \tau$ analysis can be incorporated in the standard HiggsBounds run.

\section{Validation}

Besides the nearly model-independent limits, CMS has also presented model-specific interpretations of their search results. This has been done for the MSSM, employing the benchmark scenarios proposed in Ref. [26] (see also Ref. [27]). Here, we validate our likelihood implementation in HiggsBounds against the CMS results for three of these scenarios: The $m_{h}^{\max }$, the light stop and the low$M_{H}$ scenarios (see Ref. [26] for details). The comparison of the reconstructed $95 \%$ C.L. exclusion line with the official CMS result provides a non-trivial test of our implementation: Firstly, it checks whether the exclusion likelihood agrees over a wide range of different compositions of the gluon fusion and $b$ quark associated Higgs production rates obtained in the MSSM parameter space, which are mapped onto the twodimensional likelihood grids (for fixed Higgs mass) in our reconstruction. Secondly, it tests whether our simple criterion of combining signal rates of Higgs bosons which have similar masses (overlapping within $20 \%$ ) is a reasonable approximation. Thirdly, the validation also tests whether the results obtained from the statistical hypothesis test of a single narrow resonance model can be mapped reasonably well onto the full neutral Higgs spectrum of the MSSM (and beyond).

Some deviations can be expected at the transition between regimes with different contributing Higgs combinations. As explained above, the implementation in HiggsBounds is based on the CMS results for the single narrow resonance interpretation, and the contributions of different Higgs bosons of a considered model can only be combined if their mass differences are such that they would appear as a single resonance in the CMS search. In contrast, in the dedicated CMS analyses carried out in specific MSSM benchmark scenarios it was possible to properly combine the contributions from different Higgs bosons at any given mass constellation since these have been simulated and tested with their particular masses at every parameter point. Therefore, the dedicated CMS analysis is expected to have a higher sensitivity than the HiggsBounds implementation if multiple Higgs bosons with different masses each give a non-negligible contribution to the signal yield. Furthermore, due to the simple criterion used in HiggsBounds for including/excluding

\footnotetext{
2 Technically, allowing for finite numerical precision we check for equality within $\lesssim 1 \%$.
}

the contributions of additional Higgs bosons, the considered rates in HiggsBounds may change quite abruptly in a transition region, where the selection of the tested Higgs boson combination changes. The single resonance approximation is expected to work best when the signal can be described as a single resonance formed by one or several Higgs bosons, while contributions of other Higgs bosons besides those associated with the resonance are negligible.

For predictions in the MSSM benchmark scenarios we employ the $\left(M_{A}, \tan \beta\right)$ grids of Higgs production cross sections and branching fractions for the MSSM benchmark scenarios provided by the LHC Higgs Cross Section Working Group (LHCHXSWG) [28]. ${ }^{3}$ For the $g g \rightarrow b \bar{b}(h / H / A$ ) production process we employ Santander-matching of the 4and 5-flavor scheme (FS) cross sections [57].

The results for the $m_{h}^{\max }$ scenario in the $\left(M_{A}, \tan \beta\right)$ plane are shown in Fig. 2. ${ }^{4}$ In Fig. 2a we show the distribution of the observed exclusion likelihood, $q_{\mathrm{MSSM}}^{\mathrm{obs}}$ (in color), as obtained from HiggsBounds. The corresponding $95 \%$ C.L. exclusion limit (orange, solid contour), which fulfills $q_{\mathrm{MSSM}}^{\mathrm{obs}}=$ 5.99 , is shown together with the CMS result obtained from a dedicated analysis in this benchmark scenario [20] (green, dashed contour). As mentioned in Sect. 2, the latter is based on the full combined $7+8 \mathrm{TeV}$ dataset, whereas the exclusion information implemented in HiggsBounds is only based on the $8 \mathrm{TeV}$ dataset. However, this fact is expected to lead to only minor differences in the excluded parameter regions. As can be seen, there is very good agreement between the exclusion limit reconstructed with HiggsBounds and the CMS result. Small deviations can be observed in the low $M_{A}$ region, $M_{A} \lesssim 150 \mathrm{GeV}$, where all three neutral MSSM Higgs bosons contribute substantially to the signal yield. Here, the result reconstructed with HiggsBounds excludes a slightly smaller area of parameter space. The HiggsBounds result can thus be regarded as a conservative estimate of the actual exclusion limit.

In Fig. 2b we display, for every parameter point in the $\left(M_{A}, \tan \beta\right)$ plane, the Higgs boson or combination of Higgs bosons (cluster) that has been selected to obtain the observed exclusion likelihood by the algorithm described in Sect. 3. It

\footnotetext{
3 The LHCHXSWG cross section and branching fraction grids for the MSSM benchmark scenarios are based on the following set of tools and calculations, that we list here for completeness: HIGLU [29], SusHi [30], FeynHiggs [31-36], ggH@NNLO [37], HDECAY [38,39], Prophecy4f [40,41], bbh@NNLO(5FS) [42], bbh@NLO (4FS) [43,44], ggH NLO massive [45], ggH NNLO for scalar Higgs [46,47], ggH NNLO for pseudoscalar Higgs [48,49], EW corrections from light fermions [50,51], (N)NLO (S)QCD corrections for $h / H / A$ [52-56].

${ }^{4}$ Here, and in the following figures, we show as HiggsBounds result only the constraints obtained from the CMS $\phi \rightarrow \tau \tau$ analysis and not from the full HiggsBounds application, where all currently implemented Higgs searches from LEP, the Tevatron and the LHC are taken into account.
} 


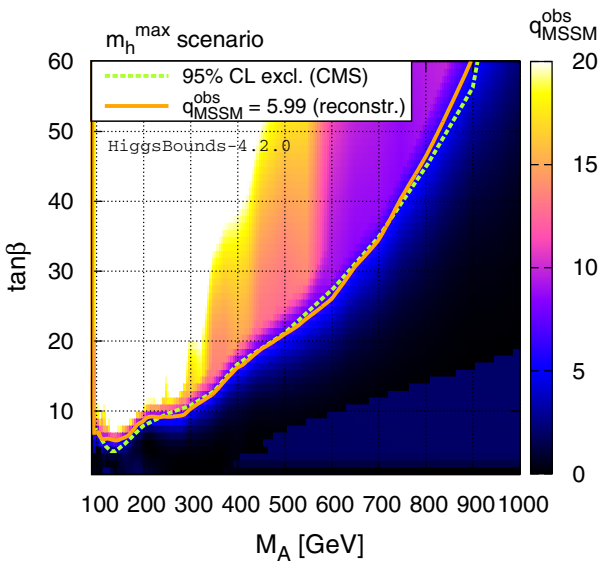

(a)

Fig. 2 Exclusion likelihood evaluated with HiggsBounds in the $\left(M_{A}, \tan \beta\right)$ plane of the MSSM $m_{h}^{\max }$ scenario. a Distribution of the observed exclusion likelihood, $q_{\mathrm{MSSM}}^{\mathrm{obs}}$, evaluated with HiggsBounds. The contours show the corresponding $95 \%$ C.L. exclusion

can be seen that all three neutral Higgs bosons are combined in most of the parameter region with $M_{A} \lesssim 160-170 \mathrm{GeV}$ and $\tan \beta \gtrsim 5$, whereas at larger $M_{A}$ values only the two heavier Higgs bosons, $H$ and $A$, which are nearly mass degenerate, are combined to yield the most sensitive constraint. At low $\tan \beta$ and large $M_{A}$ values, however, the combined signal rate of the heavier Higgs bosons becomes so small that it is instead the light Higgs boson, with mass around $120-125 \mathrm{GeV}$, that is selected to give the observed exclusion likelihood. This is because its expected exclusion likelihood is larger than that obtained for $H / A$. The observed exclusion likelihood obtained for the light Higgs boson with mass around $125 \mathrm{GeV}$ is non-zero because the best-fit point in the two-dimensional cross section grid at $m_{\phi}=125 \mathrm{GeV}$

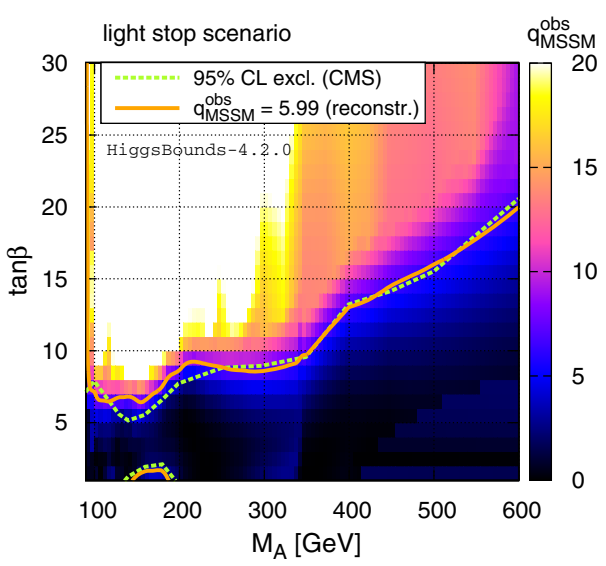

(a)

Fig. 3 Exclusion likelihood evaluated with HiggsBounds in the $\left(M_{A}, \tan \beta\right)$ plane of the MSSM light stop scenario. a Distribution of the observed exclusion likelihood, $q_{\text {MSSM }}^{\text {obs }}$, evaluated with HiggsBounds. The contours show the corresponding $95 \%$ C.L. exclusion

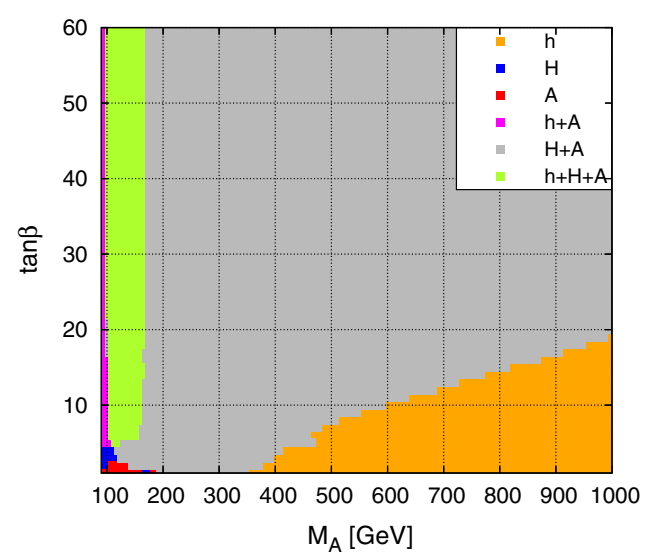

(b)

limit (orange, solid) and the CMS result obtained from a dedicated analysis in this scenario [20] (green, dashed). b Map indicating the Higgs boson or cluster of Higgs bosons with the highest sensitivity for a potential exclusion that is used for the likelihood evaluation

is not identical with the SM prediction, cf. Fig. 1a. This leads to the small, but non-zero $q_{\mathrm{MSSM}}^{\mathrm{obs}}$ values that are visible in Fig. 2a at large $M_{A}$ and small $\tan \beta$.

Next we look at the light stop benchmark scenario, for which the cross section predictions and their associated theoretical uncertainties have been discussed in detail in Ref. [58]. This scenario features a relatively low SUSY particle mass scale, $M_{\mathrm{SUSY}}=500 \mathrm{GeV}$, and large stop mixing, $X_{t}=2 M_{\text {SUSY }}$, leading to a lightest stop with a mass of $\sim 325 \mathrm{GeV}$. This leads to a reduction of the gluon fusion cross section of the light Higgs by around $10-15 \%$ with respect to the SM prediction [26]. The results of applying our exclusion likelihood implementation in this scenario are shown in Fig. 3 (with colors similar to Fig. 2). The agree-

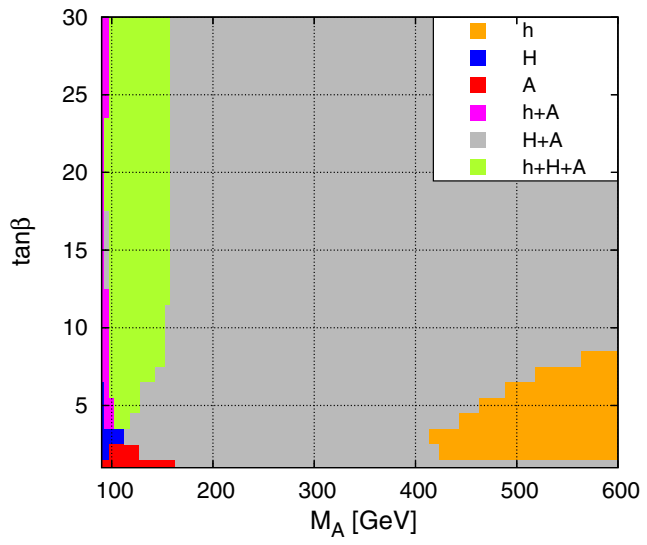

(b)

limit (orange, solid) and the CMS result obtained from a dedicated analysis in this scenario [20] (green, dashed). b Map indicating the Higgs boson or cluster of Higgs bosons with the highest sensitivity for a potential exclusion that is used for the likelihood evaluation 


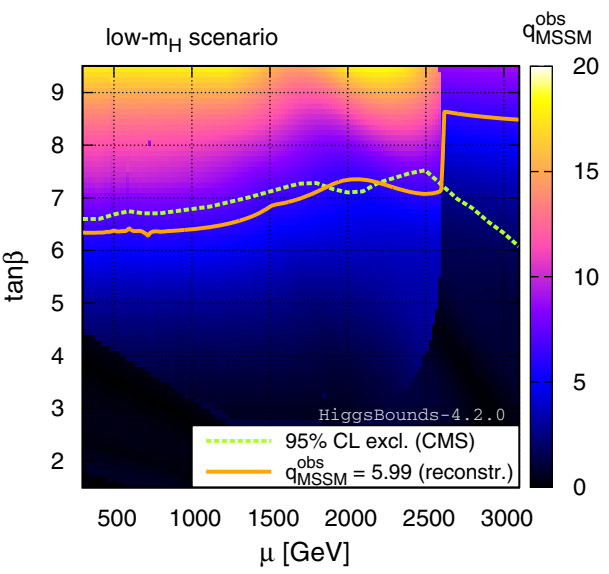

(a)

Fig. 4 Exclusion likelihood evaluated with HiggsBounds in the $\left(M_{A}, \tan \beta\right)$ plane of the MSSM low- $M_{H}$ scenario. a Distribution of the observed exclusion likelihood, $q_{\mathrm{MSSM}}^{\mathrm{obs}}$, evaluated with HiggsBounds. The contours show the corresponding 95\% C.L. exclusion limit (orange,

ment between the $95 \%$ C.L. exclusion contour obtained with HiggsBounds and the CMS result obtained from a dedicated analysis in this benchmark scenario [20], as displayed in Fig. 3a, is very good for pseudoscalar Higgs masses $M_{A} \gtrsim 250 \mathrm{GeV}$. Similarly as in the $m_{h}^{\max }$ scenario the reconstructed exclusion limit obtained from HiggsBounds is slightly weaker for lower $M_{A}$ values than the CMS result from the analysis of the benchmark scenarios. As one can see in Fig. 3b, the reconstructed likelihood in the low- $M_{A}$ region obtained from HiggsBounds is mainly based on a combination of the $H$ and $A$ signals, while in most part of this parameter region the light Higgs boson at $125 \mathrm{GeV}$ is not covered by the $20 \%$ mass overlap criterion used in Higgs Bounds. In contrast, in the dedicated CMS analysis in this scenario also the contribution from the light Higgs boson $h$ is properly combined with the contributions from the other neutral Higgs bosons. The latter analysis therefore has a slightly higher sensitivity in this region, which means that the exclusion bound that we find here is slightly conservative compared to the dedicated CMS result. In addition to the excluded parameter region at values of $\tan \beta \gtrsim 5$, the light stop scenario features an additional small excluded area at lower $\tan \beta$ values, namely $\tan \beta \lesssim 2$, and $M_{A} \sim 145-190 \mathrm{GeV}$. The exclusion contour evaluated with HiggsBounds matches very well with the CMS result also in this region, where gluon fusion is the dominant production mode.

Finally, we test our implementation against the results obtained in the low- $M_{H}$ scenario, where the heavy $\mathcal{C P}$-even Higgs boson is interpreted as the discovered SM-like Higgs boson at around $\sim 125 \mathrm{GeV}$ and the light $\mathcal{C P}$-even Higgs is largely decoupled from the SM gauge bosons [59]. Unlike the other benchmark scenarios, which use $M_{A}$ as a free parameter, this scenario is defined as a two-dimensional parameter

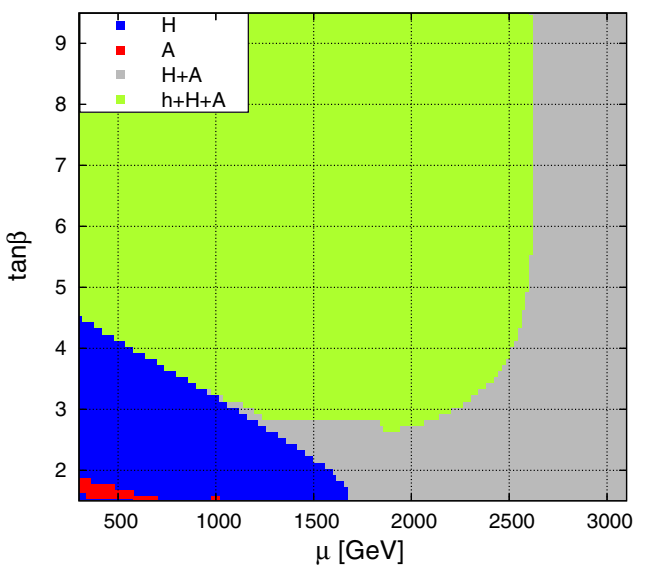

(b)

solid) and the CMS result obtained from a dedicated analysis in this scenario [20] (green, dashed). b Map indicating the Higgs boson or cluster of Higgs bosons with the highest sensitivity for a potential exclusion that is used for the likelihood evaluation

plane in $\tan \beta$ and the Higgsino mixing parameter $\mu$. The mass of the pseudoscalar Higgs, $M_{A}$, is fixed to $110 \mathrm{GeV}$, which leads to a lightest Higgs mass that varies mostly between $\sim 80$ and $\sim 105 \mathrm{GeV}$, but reaching even lower values at very low $\tan \beta$ and very high $\mu$. Since in the MSSM a low value of $M_{A}$ implies also a light charged Higgs boson, this scenario served in particular as a benchmark for the LHC searches for charged Higgs bosons in top quark decays. In fact, the parameter space for this scenario in the MSSM is meanwhile essentially excluded [60-62] (see also Ref. [63]). Nevertheless this benchmark scenario is still very useful for our validation since all three neutral Higgs bosons have similar masses and thus contribute non-trivially to the analysis.

The comparison of the exclusion limits that have been reconstructed with HiggsBounds with the CMS results is shown in Fig. 4. It can be observed in Fig. 4a that there is rather good agreement between the exclusion limit obtained with HiggsBounds and the CMS result for $\mu$ values up to $\mu \sim 2600 \mathrm{GeV}$. At this value of $\mu$ (depending on $\tan \beta$ ) the reconstructed exclusion limit develops an "edge", and for higher $\mu$ values the reconstructed exclusion limit is significantly weaker than the one of the CMS result. The reason for this behavior is that at large $\mu$ the light Higgs mass becomes smaller than $88 \mathrm{GeV}$ and is hence not combined with the heavier Higgs bosons $A$ and $H$ in HiggsBounds. This can be seen in Fig. 4b. In this parameter region, the tested signal rate is therefore significantly smaller than in the case of a full combination of $h, H$ and $A$, and the resulting exclusion limit is accordingly weaker. In contrast, in the CMS analysis the signal yield of the light Higgs $h$ has been properly taken into account even for very low mass values, and the possibly decreasing signal efficiency is partially compensated by the increasing production cross section, leading to the sig- 
nificantly stronger exclusion at high $\mu$ values obtained by CMS. $^{5}$

In almost all the remaining parameter space, all three Higgs bosons are combined by HiggsBounds, as can be seen in Fig. 4b, and the reconstructed exclusion contour resembles the CMS result for $\mu$ values below $\mu \sim$ $2600 \mathrm{GeV}$. The slight deviations observed could result from mass-dependent selection efficiencies for the $h$ and $H$ signal yields, which cannot be accounted for in the HiggsBounds implementation since this information is not publicly available. Overall, even for this rather extreme scenario in the MSSM we find that the exclusion likelihood reconstructed with HiggsBounds approximates the results of a dedicated analysis reasonably well for large parts of the parameter space.

\section{Example application: "Alignment without decoupling"}

We now go beyond the validation with official CMS results and illustrate the usefulness of our exclusion likelihood implementation for another MSSM scenario. We consider here a scenario where the couplings of the light $\mathcal{C P}$-even Higgs boson become SM-like for a certain range of $\tan \beta$ values, independently of the masses of the remaining Higgs spectrum. The existence of this so-called alignment limit was first pointed out in Ref. [64] for the 2HDM. After the Higgs discovery this possibility has gained attention through a series of papers [65-69], see also the " $\tau$-phobic" benchmark scenario in Ref. [26]. In the MSSM the alignment limit can be realized independently of the decoupling of the heavier Higgs states through a cancellation between tree-level and higher-order contributions in the Higgs sector. This cancellation can occur at relatively large values of $\tan \beta$ and $\mu \gtrsim M_{S}$, with $M_{S}=\sqrt{m_{\tilde{t}_{1}} m_{\tilde{t}_{2}}}$ being the stop mass scale. In the approximation $\tan \beta \gg 1$, and taking into account for simplicity only the dominant corrections at one loop, the alignment condition reads [69]

$\tan \beta=\frac{M_{h}^{2}+M_{Z}^{2}+\frac{3 m_{t}^{4} \mu^{2}}{4 \pi^{2} v^{2} M_{S}^{2}}\left(\frac{A_{t}^{2}}{2 M_{S}^{2}}-1\right)}{\frac{3 m_{t}^{4} \mu A_{t}}{4 \pi^{2} v^{2} M_{S}^{2}}\left(\frac{A_{t}^{2}}{6 M_{S}^{2}}-1\right)}$.

\footnotetext{
5 A better agreement in the large $\mu$ parameter region could be obtained by increasing the mass overlap value of $20 \%$ in the criterion for forming Higgs boson combinations to a sufficiently high value. However, firstly, there is no strong physics motivation to choose a value well beyond the quoted mass resolution of $\sim 20 \%$ of the experimental $\tau \tau$ analysis. Secondly, values larger than $20 \%$ might lead to too aggressive exclusions in some scenarios. We therefore stick to the value of $20 \%$ as the default setting.
}

Here, $M_{Z}$ and $m_{t}$ are the $Z$ boson and top quark mass, respectively. $M_{h}$ denotes the light $\mathcal{C P}$-even Higgs boson mass in the above approximation, and $v \approx 246 \mathrm{GeV} . A_{t}$ is the trilinear soft-breaking term in the stop sector.

Solutions of Eq. (12) with $\tan \beta>0$ exist if $\mu A_{t}\left(A_{t}^{2}-\right.$ $\left.6 M_{S}^{2}\right)>0$. Typically, in order to achieve the correct Higgs mass $M_{h} \sim 125 \mathrm{GeV}$ for not too large values of the stop masses, the stop mixing is chosen in the region where the prediction for $M_{h}$ is maximized, i.e. $\left|X_{t}\right| \sim\left|A_{t}\right| \sim \pm \sqrt{6} M_{S}$ (at one-loop). Therefore, for $\mu A_{t}>0\left(\mu A_{t}<0\right)$, the alignment condition has viable solutions for values of $\left|A_{t}\right|$ that are slightly above (below) the value where the prediction for $M_{h}$ is maximized. By increasing $\left|\mu A_{t} / M_{S}^{2}\right|$ it is possible to lower the $\tan \beta$ value at which alignment occurs. An MSSM benchmark scenario of this kind for BSM Higgs searches at the LHC has recently been proposed in Ref. [69].

Here, we investigate the benchmark scenario proposed in Ref. [69], which is essentially a modification of the $m_{h}^{\bmod +}$ scenario [26] to allow for alignment independent of decoupling. This so-called $m_{h}^{\text {alt }}$ scenario is defined by the parameter values (in the on-shell scheme)

$M_{1}=100 \mathrm{GeV}, \quad M_{2}=200 \mathrm{GeV}, \quad M_{3}=1500 \mathrm{GeV}$,

$m_{\tilde{\ell}}=m_{\tilde{q}} \equiv M_{Q}, \quad A_{\ell}=A_{q} \equiv A_{t}, \quad A_{t} / M_{Q}=2.45$.

In contrast to the benchmark scenarios of Ref. [26], the parameters $\mu$ and $M_{Q}$ are adjustable parameters in the $m_{h}^{\text {alt }}$ scenario. For convenience, the slepton, sbottom and first and second generation squark soft-breaking mass parameters are set to $M_{Q}$, however, these can easily be adjusted to higher values in order to avoid constraints from SUSY searches at the LHC as their influence on the Higgs phenomenology is negligible here. We follow the suggestion to set $M_{Q}$ to $1 \mathrm{TeV}$ per default and, if necessary, increase this value until a light Higgs mass of $m_{h} \geq 123 \mathrm{GeV}$ is obtained. In practice, this is only relevant at very low values of $\tan \beta$ in the benchmark scenario defined by Eq. (13). The parameter $\mu$ is then adjusted according to a chosen ratio $\mu / M_{Q}$. We focus here on the choice $\mu / M_{Q}=3$, implying rather large values of $\mu$, where alignment independent of decoupling occurs at $\tan \beta \sim 10$.

The MSSM predictions are obtained using the public computer codes FeynHiggs-2 .10.2 [31-34,36] for the Higgs masses, couplings and branching fractions, and SusHi-1 . 4 .1 [30] for the gluon fusion and $b$ quark associated production cross sections of the three neutral Higgs bosons.

The numerical results for this benchmark scenario are displayed in Fig. 5. The observed exclusion likelihood from the CMS $\phi \rightarrow \tau \tau$ search as obtained from HiggsBounds is shown in color in Fig. 5a, and the orange contour indicates the resulting observed $95 \%$ C.L. exclusion line. For com- 


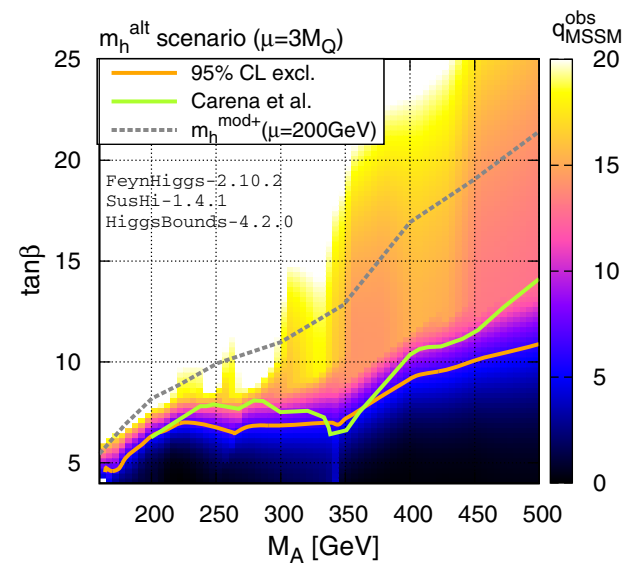

(a)

Fig. 5 Constraints from LHC Higgs searches in the alignment benchmark scenario $m_{h}^{\text {alt }}$ (with $\mu=3 M_{Q}$ ): a Distribution of the exclusion likelihood from the CMS $\phi \rightarrow \tau \tau$ search and observed $95 \%$ C.L. exclusion line as obtained from HiggsBounds. For comparison, also the corresponding $95 \%$ C.L. exclusion line given in Ref. [69] (green, solid) and the $95 \%$ C.L. exclusion line in the $m_{h}^{\bmod +}$ scenario with

parison, the green contour shows the exclusion line obtained in Ref. [69] using results from the same CMS analysis, however, following a more simplistic approach. ${ }^{6}$ As can be seen from the figure, the more advanced implementation of the observed exclusion likelihood in HiggsBounds leads to a somewhat stronger $95 \%$ C.L. exclusion limit over most of the parameter space. The relative behavior seems to be different in the $t \bar{t}$ threshold region, $M_{A} \approx 2 m_{t} \approx 345 \mathrm{GeV}$, where in particular the $g g \rightarrow A$ cross section is enhanced. However, the approximations made in Ref. [69] appear to be least reliable in this region. The gray dotted line shows the exclusion limit obtained by CMS for the $m_{h}^{\bmod +}$ scenario. As discussed in Ref. [26], the excluded regions in the benchmark scenarios are significantly affected if decay modes of the heavy Higgs bosons $H$ and $A$ into SUSY particles are kinematically open and unsuppressed. The presence of such decay modes leads to a sizable reduction of the $H / A \rightarrow \tau \tau$ branching fractions and therefore to a smaller excluded region. In the alignment scenario $\mu$ is very large, leading to a negligible Higgsino component in the light neutralinos and chargino. The branching fractions for the Higgs decays to neutralinos and charginos are therefore essentially absent. In addition, the heavy Higgs decays to gauge bosons, $H \rightarrow W^{+} W^{-}$and $H \rightarrow Z Z$, are also suppressed, as the responsible coupling $\alpha \cos (\beta-\alpha)$ vanishes in the alignment limit. As a result, the

\footnotetext{
6 The limit in Ref. [69] has been obtained by "reverse-engineering" an inclusive $[\sigma(g g \rightarrow \phi)+\sigma(g g \rightarrow b \bar{b} \phi)] \times \mathrm{BR}(\phi \rightarrow \tau \tau)$ limit from the CMS results for the $m_{h}^{\bmod +}$ scenario with $\mu=200 \mathrm{GeV}$ [26], and applying this cross section limit to the given alignment benchmark scenario. In particular, this approximation does not take into account the sensitivity of the limit on the individual contributions from the gluon fusion and $b$ quark associated Higgs production processes.
}

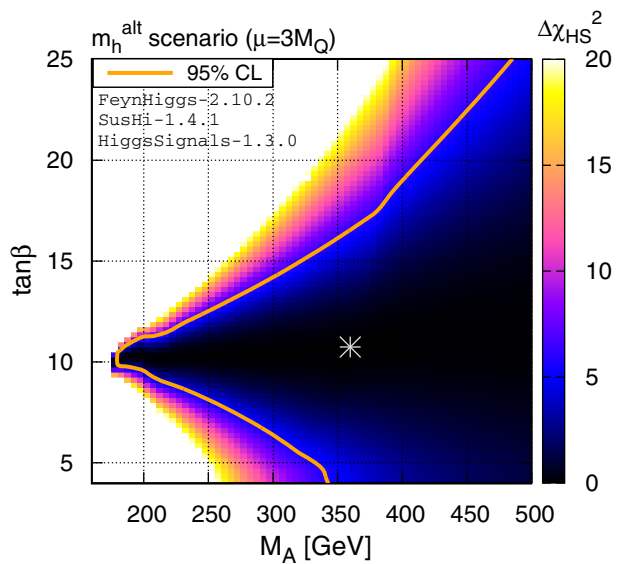

(b)

$\mu=200 \mathrm{GeV}$ obtained from HiggsBounds (gray, dashed) are shown. b Likelihood distribution, $\Delta \chi_{\mathrm{HS}}^{2}$, obtained from testing the signal rates of the light Higgs boson $h$ against a combination of Higgs rate measurements from the Tevatron and LHC experiments, obtained with HiggsSignals. The minimal $\chi^{2}$ is found at the gray asterisk

$H / A \rightarrow \tau \tau$ branching fraction is significantly higher in the alignment scenario than in the $m_{h}^{\bmod +}$ scenario, which leads to a much larger excluded region in the alignment scenario, see also the discussion in Ref. [69].

In order to illustrate the complementarity between the constraints from the CMS $\phi \rightarrow \tau \tau$ search and the constraints obtained from the signal rate measurements of the discovered Higgs boson, we show in Fig. 5b the likelihood distribution, $\Delta \chi_{\mathrm{HS}}^{2}$, obtained from a $\chi^{2}$ test of the light Higgs boson signal rates against a combination of the latest rate measurements from the LHC [70-78] and the Tevatron [79,80], using the public computer code HiggsSignals-1.3 .0 [13] (see also Refs. [19,81]). The 95 \% C.L. preferred region lies within the orange contours in Fig. 5b. It is given by the $\chi^{2}$ difference with respect to the minimal $\chi^{2}$ value (located in the alignment region and indicated as gray asterisk in Fig. 5b), $\Delta \chi_{\mathrm{HS}}^{2} \equiv \chi_{\mathrm{HS}}^{2}-\chi_{\mathrm{HS} \text {, min }}^{2} \leq 5.99$. It can be seen that the $\chi^{2}$ distribution becomes independent of $M_{A}$ at around $\tan \beta \approx 10$, indicating that the couplings of the light Higgs become SMlike independently of the decoupling of the heavier Higgs states.

Since we now have the exclusion likelihood $q_{\mathrm{MSSM}}^{\mathrm{obs}}$ from the CMS $\phi \rightarrow \tau \tau$ search available, we can perform a statistical combination with the constraints from the Higgs rate measurements by constructing the global $\chi^{2}$ function $\chi_{\mathrm{tot}}^{2}=q_{\mathrm{MSSM}}^{\mathrm{obs}}+\chi_{\mathrm{HS}}^{2}$. The resulting $\Delta \chi_{\mathrm{tot}}^{2}$ distribution ${ }^{7}$ is shown in Fig. 6 . The constraints from the $\phi \rightarrow \tau \tau$ searches at the LHC are highly complementary to the rate measure-

\footnotetext{
$\overline{7 \text { Again, } \Delta \chi_{\text {tot }}^{2}}$ is the $\chi^{2}$ difference with respect to the minimal $\chi^{2}$ value (obtained at $M_{A}=500 \mathrm{GeV}, \tan \beta=4$, i.e. in the lower right corner of Fig. 6), now based on the global likelihood $\chi_{\text {tot }}^{2}$.
} 


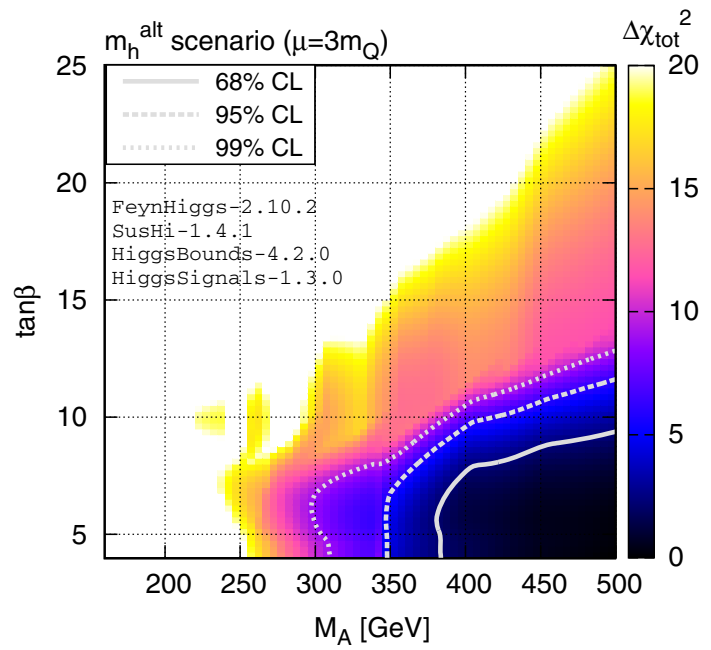

Fig. 6 Combination of constraints from the CMS $\phi \rightarrow \tau \tau$ search and the latest Higgs rate measurements in the MSSM alignment scenario (with $\mu=3 M_{Q}$ ): The global $\chi^{2}$ function, $\Delta \chi_{\text {tot }}^{2}$, based on the likelihoods provided by HiggsBounds and HiggsSignals, is shown in color; The contours indicate the $1 \sigma, 2 \sigma$ and $3 \sigma$ allowed regions

ments, since they are particularly sensitive at higher values of $\tan \beta$ where the production process $g g \rightarrow b \bar{b} \phi$ is enhanced. In the $m_{h}^{\text {alt }}$ scenario with $\mu=3 M_{Q}$, the combination of both constraints yields a lower limit of $M_{A} \gtrsim 350 \mathrm{GeV}$ at the $95 \%$ C.L. Thus, alignment of the light Higgs boson occurring without the simultaneous decoupling of the heavier Higgs states is ruled out for this scenario. The alignment without decoupling limit can be pushed to lower values of $\tan \beta$ in this scenario, where the constraints from the $\phi \rightarrow \tau \tau$ searches are less significant, only by choosing even more extreme values of $\mu A_{t} / M_{Q}^{2}$, which potentially leads to problems with vacuum stability $[82,83]$.

\section{Conclusions}

LHC searches for non-standard Higgs bosons decaying into tau lepton pairs constitute a sensitive experimental probe for BSM physics. Recently, the CMS collaboration published the likelihood information for their Higgs boson searches in the $\tau^{+} \tau^{-}$final state [20]. The likelihood is given as a function of the two relevant Higgs production channels, gluon fusion and $b$ quark associated production, for various mass values of the narrow resonance assumed for the signal model. In this paper we have shown how this experimental information can be utilized to test large classes of theoretical models. In particular, we have developed a simple algorithm that maps an arbitrary model with multiple neutral Higgs bosons onto a model with a single narrow resonance, for which the corresponding exclusion likelihood from the CMS search can be determined. We have described the inclusion of this method into the new version of the publicly available For- tran code HiggsBounds (version 4.2.0 and higher). For nearly any model under consideration, HiggsBounds provides an evaluation of the exclusion likelihood for a model parameter point based on the information from Ref. [20]. Similarly, if requested, HiggsBounds can also perform a test of whether or not a given parameter point is excluded at the $95 \%$ C.L. based on all available searches, including the new $\tau^{+} \tau^{-}$result. The approach to test BSM models with exclusion limits is complementary to testing the compatibility of a given model with the observed Higgs signal (and possible future signals of additional Higgs bosons). The latter kind of information is contained in the sister program HiggsSignals, and both programs can be used together in order to obtain the combined likelihood information from the search limits and the Higgs rate measurements. Both codes are available at http://higgsbounds.hepforge.org.

We have validated our implementation of the $\tau^{+} \tau^{-}$search results into HiggsBounds by comparing the $95 \%$ C.L. exclusion contours obtained with HiggsBounds with the ones obtained by CMS from dedicated analyses in three Higgs benchmark scenarios [26] in the MSSM. We found very good agreement in the parameter regions where the sensitivity of the search is dominated by a single combination of Higgs bosons that can be identified with a single narrow resonance assuming an experimental mass resolution of $20 \%$. As expected, the largest but still relatively small deviations occur in parameter regions where all neutral MSSM Higgs bosons are relatively close in mass and contribute comparably to the signal yield.

As an application, we have discussed the combined constraints of the $\tau \tau$ search and the rate measurements of the SM-like Higgs at $125 \mathrm{GeV}$ in a recently proposed MSSM benchmark scenario, where the lightest Higgs boson obtains SM-like couplings independently of the decoupling of the heavier Higgs states. Here we combined the $\chi^{2}$ analysis of the rate measurements for the Higgs signal, evaluated with HiggsSignals, with the exclusion likelihood from the non-observation in the $\tau^{+} \tau^{-}$search channel, evaluated with HiggsBounds. We have shown that the combined information yields very significant constraints on the available parameter space in this scenario and in fact disfavors the "alignment without decoupling region" in the studied benchmark model.

We encourage ATLAS and CMS to continue providing their search results including the relevant likelihood information. This will greatly facilitate the application of the search results for testing BSM models.

Acknowledgments We thank Felix Frensch, Andrew Gilbert, Howie Haber, Sasha Nikitenko, Alexei Raspereza and Roger Wolf for helpful discussions. In particular, we are grateful to Felix Frensch, Andrew Gilbert and Roger Wolf for communication regarding the CMS results of Ref. [20] and for a careful reading of our manuscript. This work has been supported by the Collaborative Research Center SFB676 of the DFG, 
"Particles, Strings and the early Universe", and in part by the European Commission through the "HiggsTools" Initial Training Network PITNGA-2012-316704. The work of S.H. is supported in part by CICYT (Grant FPA 2013-40715-P) and by the Spanish MICINN's ConsoliderIngenio 2010 Program under Grant MultiDark No. CSD2009-00064. T.S. is supported in part by U.S. Department of Energy Grant Number DE-FG02-04ER41286, and in part by a Feodor-Lynen research fellowship sponsored by the Alexander von Humboldt Foundation.

Open Access This article is distributed under the terms of the Creative Commons Attribution 4.0 International License (http://creativecomm ons.org/licenses/by/4.0/), which permits unrestricted use, distribution, and reproduction in any medium, provided you give appropriate credit to the original author(s) and the source, provide a link to the Creative Commons license, and indicate if changes were made.

Funded by $\mathrm{SCOAP}^{3}$.

\section{Appendix: User guide: how to obtain the exclusion like- lihood with HiggsBounds}

The exclusion likelihood information for the CMS $\phi \rightarrow \tau \tau$ analysis [20] is implemented in HiggsBounds from version 4.2 on. As described in Sect. 3 this information is used in a standard HiggsBounds run to reconstruct the expected and observed $95 \%$ C.L. exclusion limit, which is then considered alongside all other available Higgs search limits in the full HiggsBounds application. This leads to the global information whether the tested parameter point is allowed or excluded at the $95 \%$ C.L. Beyond this information the value for the exclusion likelihood for the model parameter point under investigation, $q_{\text {model }}$, can also be obtained directly via HiggsBounds Fortran subroutines, enabling the user to incorporate this information e.g. in a global parameter fit. In the following we document the relevant subroutines that make this information accessible.

The main routine that runs the algorithm presented in Sect. 3 to obtain the exclusion likelihood is:

HiggsBounds_get_likelihood(int analysisID, int Hindex, int $\mathrm{nc}$, int cbin, dble $\mathrm{M}$, dble 1lh, $\operatorname{char}(*)$ obspred)

The (mandatory) input argument ${ }^{8}$ analysisID specifies the analysis for which the likelihood should be obtained. At the moment, the CMS $\phi \rightarrow \tau \tau$ analysis based on the full $8 \mathrm{TeV}$ dataset (analysisID = 3316) is the only available likelihood, but the framework is easily extendable for future experimental results. The output values provide information about the selected Higgs boson combination (or Higgs cluster):

- Hindex gives the index $i$ of the Higgs boson $h_{i}$, which provided the initial seed to form the dominant Higgs cluster (cf. Sect. 3, item 1),

\footnotetext{
${ }^{8}$ Here, and in the following, input arguments and optional input arguments are highlighted in dark blue and green, respectively. The remaining arguments are output values.
}

- nc gives the number of Higgs bosons contained in the combination,

- cbin is a binary code (bitmask) for the identifiers of the participating Higgs bosons. The binary code is given by summing over $2^{(i-1)}$ for all involved Higgs bosons. ${ }^{9}$ For example, in the MSSM (with common indexing $h_{1}=h$, $h_{2}=H, h_{3}=A$ ), the combination $H+A$ would give cbin $=6$, whereas a cluster formed only by the light Higgs $h$ gives cbin $=1$.

The output value $\mathrm{M}$ gives the averaged mass value, calculated according to Eq. (8), at which the likelihood value has been evaluated. The computed value of the likelihood, $q_{\text {model }}$, is returned as $11 \mathrm{~h}$. The final argument is an optional input, obspred, which takes a string value that can be either 'obs' or 'pred', specifying whether the observed or expected (or predicted) exclusion likelihood should be evaluated, respectively. The default behavior if this argument is not provided is that the routine returns the observed likelihood, following the algorithm described in Sect. 3.

In addition to the main subroutine we provide the following two auxiliary routines, which may be helpful to understand the obtained results.

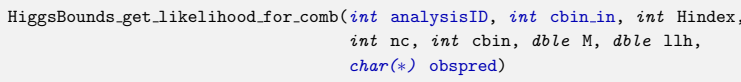

This routine evaluates the likelihood for a specific selection of Higgs bosons that should be considered for the test. Higgs bosons that are not available for a possible formation of a Higgs cluster are specified with the input parameter cbin_in, which is a binary code following the same convention as cbin above. The remaining arguments are the same as above, with the only exception that obspred is now a mandatory input parameter. Among the available Higgs bosons, the routine selects the Higgs combination with the maximal likelihood value and provides the corresponding results.

HiggsBounds_get_likelihood_for_Higgs(int analysisID, int cbin_in, int Hindex, int $\mathrm{nc}$, int cbin, dble $\mathrm{M}$, dble $1 \mathrm{lh}$, $\operatorname{char}(*)$ obspred)

This auxilliary subroutine works in a similar way as above. However, the additional input argument Hindex forces the routine to consider only Higgs clusters that contain the specified Higgs boson $h_{i}$.

In global parameter fits, where both the conventional HiggsBounds output (95\% C.L. exclusion) as well as the likelihood information is used, it is often convenient to deactivate specific analyses during the standard HiggsBounds

9 The indexing of the Higgs bosons is identical to the ordering in which the user chooses to specify the theoretical input for HiggsBounds [9, $10,12]$. 
Table 1 Implemented $95 \%$ C.L. exclusion limits from LHC searches for BSM Higgs bosons with $\tau \tau$ final states in HiggsBounds -4.2 . The analysisID is used as a unique identifier for an individual analysis and can be used to deactivate/activate them in HiggsBounds (see text)

\begin{tabular}{lllll}
\hline analysisID & Experiment & Luminosity and CM-Energy & Additional notes & References \\
\hline 3316 & CMS & $19.7 \mathrm{fb}^{-1}$ at $8 \mathrm{TeV}$ & Using $-2 \ln L$ reconstruction & {$[20]$} \\
2014049 & ATLAS & $19.5-20.3 \mathrm{fb}^{-1}$ at $8 \mathrm{TeV}$ & Profiled limit on $g g \rightarrow b \bar{b} \phi$ process \\
20140492 & ATLAS & $19.5-20.3 \mathrm{fb}^{-1}$ at $8 \mathrm{TeV}$ & Profiled limit on $g g \rightarrow \phi$ process & {$[21]$} \\
\hline
\end{tabular}

run, since these are better described by the likelihood information. In particular, the $95 \%$ C.L. limits from previous $\mathrm{BSM} \phi \rightarrow \tau \tau$ searches can be deactivated if instead the CMS $\phi \rightarrow \tau \tau$ exclusion likelihood is used. In order to do so, we provide two new subroutines, contained in the Fortran module 'channels'.

HiggsBounds_deactivate_analyses(int(:) analysisID_list)

This routine should be called before the subroutine run_higgsbounds in order to deactivate the analyses specified by the integer array analysisid_list. for convenience, the analysis identifiers of the currently implemented lhc $\phi \rightarrow \tau \tau$ searches are given in Table 1 .

HiggsBounds_activate_all_analyses()

This subroutine can be used at any time to re-activate all previously deactivated analyses for the succeeding HiggsBounds run.

In order to demonstrate the use of these subroutines, we provide the example program HBwithLHClikelihood, included in the / example_programs / directory of the HiggsBounds distribution. This program shows how to obtain the observed exclusion likelihood from the CMS $\phi \rightarrow \tau \tau$ analysis in the MSSM $m_{h}^{\max }$ scenario, such that the user should be able to directly reproduce Fig. 2 . The example can be compiled by calling 'make HBwithLHClikelihood' in the HiggsBounds main folder, and run from the example_programs folder by calling '. / HBwithLHClikelihood'. Following a successful run, the gnuplot scripts 'plot_mhmax_llh.gnu' and 'plot_ mhmax_llh_comb.gnu' in the same folder then reproduce Fig. 2.

\section{References}

1. F. Englert, R. Brout, Phys. Rev. Lett. 13, 321-323 (1964)

2. P.W. Higgs, Phys. Lett. 12, 132-133 (1964)

3. P.W. Higgs, Phys. Rev. Lett. 13, 508-509 (1964)

4. G. Guralnik, C. Hagen, T. Kibble, Phys. Rev. Lett. 13, 585-587 (1964)

5. P.W. Higgs, Phys. Rev. 145, 1156-1163 (1966)

6. T. Kibble, Phys. Rev. 155, 1554-1561 (1967)
7. ATLAS collaboration, G. Aad et. al. Phys.Lett. B 716, 1-29 (2012). arXiv: 1207.7214

8. CMS collaboration, S. Chatrchyan et. al., Phys. Lett. B 716, 30 61(2012) . arXiv:1207.7235

9. P. Bechtle, O. Brein, S. Heinemeyer, G. Weiglein, K.E. Williams, Comput. Phys. Commun. 181, 138-167 (2010). arXiv:0811.4169

10. P. Bechtle, O. Brein, S. Heinemeyer, G. Weiglein, K.E. Williams, Comput. Phys. Commun. 182, 2605-2631 (2011). arXiv: 1102.1898

11. P. Bechtle, O. Brein, S. Heinemeyer, O. Stål, T. Stefaniak, et. al., PoS CHARGED2012, 024 (2012). arXiv:1301.2345

12. P. Bechtle, O. Brein, S. Heinemeyer, O. Stål, T. Stefaniak, et. al., Eur. Phys. J. C 74(3), 2693 (2014). arXiv:1311.0055

13. P. Bechtle, S. Heinemeyer, O. Stål, T. Stefaniak, G. Weiglein, Eur. Phys. J. C 74(2), 2711 (2014). arXiv:1305.1933

14. K. de Vries, E. Bagnaschi, O. Buchmueller, R. Cavanaugh, M. Citron, et. al., arXiv: 1504.0326

15. P. Bechtle, S. Heinemeyer, O. Stål, T. Stefaniak, G. Weiglein, et. al., Eur. Phys. J. C 73(4) 2354 (2013). arXiv:1211.1955

16. P. Bechtle, T. Bringmann, K. Desch, H. Dreiner, M. Hamer et al., JHEP 1206, 098 (2012). arXiv: 1204.4199

17. P. Bechtle, K. Desch, H. K. Dreiner, M. Hamer, M. Krämer, et. al., PoS EPS-HEP2013, 313 (2013). arXiv:1310.3045

18. P. Bechtle, K. Desch, H. K. Dreiner, M. Hamer, M. Krämer, et. al., arXiv: 1410.6035

19. P. Bechtle, S. Heinemeyer, O. Stål, T. Stefaniak, G. Weiglein, JHEP 1411, 039 (2014). arXiv: 1403.1582

20. CMS collaboration, V. Khachatryan et. al., JHEP 1410, 160 (2014). arXiv:1408.3316. The two-dimensional $-2 \ln \mathcal{L}$ results for the narrow resonance model can be obtained from:https://twiki.cern.ch/ twiki/bin/view/CMSPublic/Hig13021PaperTwiki

21. ATLAS collaboration, G. Aad et. al., JHEP 1411, 056 (2014). arXiv: 1409.6064

22. The ATLAS Collaboration, The CMS Collaboration, The LHC Higgs Combination Group collaboration Tech. Rep. CMS-NOTE2011-005. ATL-PHYS-PUB-2011-11, CERN, Geneva (2011)

23. E. Fuchs, S. Thewes, G. Weiglein, Eur. Phys. J. C 75(6), 254 (2015). arXiv: 1411.4652

24. E. Fuchs, arXiv: 1411.5239

25. E. Fuchs. PhD thesis, 2015

26. M. Carena, S. Heinemeyer, O. Stål, C. Wagner, G. Weiglein Eur. Phys. J. C 73(9), 2552 (2013). arXiv:1302.7033

27. LHC Higgs Cross Section Working Group collaboration, S. Heinemeyeret. al., arXiv:1307.1347

28. LHC Higgs Cross Section Working Group, https://twiki.cern. ch/twiki/bin/view/LHCPhysics/LHCHXSWGMSSMNeutral. Accessed Nov 2014

29. M. Spira, arXiv:hep-ph/9510347

30. R.V. Harlander, S. Liebler, H. Mantler, Comput. Phys. Commun. 184, 1605-1617 (2013). arXiv:1212.3249

31. S. Heinemeyer, W. Hollik, G. Weiglein, Comput. Phys. Commun. 124, 76-89 (2000). arXiv:hep-ph/9812320

32. S. Heinemeyer, W. Hollik, G. Weiglein, Eur. Phys. J. C 9, 343-366 (1999). arXiv:hep-ph/9812472 
33. G. Degrassi, S. Heinemeyer, W. Hollik, P. Slavich, G. Weiglein, Eur. Phys. J. C 28, 133-143 (2003). arXiv:hep-ph/0212020

34. M. Frank, T. Hahn, S. Heinemeyer, W. Hollik, H. Rzehak et al., JHEP 0702, 047 (2007). arXiv:hep-ph/0611326

35. T. Hahn, S. Heinemeyer, W. Hollik, H. Rzehak, G. Weiglein, Comput. Phys. Commun. 180, 1426-1427 (2009)

36. T. Hahn, S. Heinemeyer, W. Hollik, H. Rzehak, G. Weiglein, Phys. Rev. Lett. 112(14), 141801 (2014). arXiv:1312.4937

37. R.V. Harlander, W.B. Kilgore, Phys. Rev. Lett. 88, 201801 (2002). arXiv:hep-ph/0201206

38. A. Djouadi, J. Kalinowski, M. Spira, Comput. Phys. Commun. 108, 56-74 (1998). arXiv:hep-ph/9704448

39. A. Djouadi, M.M. Muhlleitner, M. Spira, Acta Phys. Polon. B 38, 635-644 (2007). arXiv:hep-ph/0609292

40. A. Bredenstein, A. Denner, S. Dittmaier, M.M. Weber, Phys. Rev. D 74, 013004 (2006). arXiv:hep-ph/0604011

41. A. Bredenstein, A. Denner, S. Dittmaier, M.M. Weber, JHEP 02, 080 (2007). arXiv:hep-ph/0611234

42. R.V. Harlander, W.B. Kilgore, Phys. Rev. D 68, 013001 (2003). arXiv:hep-ph/0304035

43. S. Dittmaier, M. Krämer, M. Spira, Phys. Rev. D 70, 074010 (2004). arXiv:hep-ph/0309204

44. S. Dawson, C. Jackson, L. Reina, D. Wackeroth, Phys. Rev. D 69 , 074027 (2004). arXiv:hep-ph/0311067

45. M. Spira, A. Djouadi, D. Graudenz, P. Zerwas, Nucl. Phys. B 453, 17-82 (1995). arXiv:hep-ph/9504378

46. C. Anastasiou, K. Melnikov, Nucl. Phys. B 646, 220-256 (2002). arXiv:hep-ph/0207004

47. V. Ravindran, J. Smith, W.L. van Neerven, Nucl. Phys. B 665 , 325-366 (2003). arXiv:hep-ph/0302135

48. R. V. Harlander, W.B. Kilgore, JHEP 0210, 017 (2002). arXiv:hep-ph/0208096

49. C. Anastasiou, K. Melnikov, Phys. Rev. D 67, 037501 (2003). arXiv:hep-ph/0208115

50. U. Aglietti, R. Bonciani, G. Degrassi, A. Vicini, Phys. Lett. B 595, 432-441 (2004). arXiv:hep-ph/0404071

51. R. Bonciani, G. Degrassi, A. Vicini, Comput. Phys. Commun. 182, 1253-1264 (2011). arXiv:1007.1891

52. R. V. Harlander, M. Steinhauser, JHEP 0409, 066 (2004). arXiv:hep-ph/0409010

53. R. Harlander, P. Kant, JHEP textbf0512, 015 (2005). arXiv:hep-ph/0509189

54. G. Degrassi, P. Slavich, JHEP 1011, 044 (2010). arXiv:1007.3465

55. G. Degrassi, S. Di Vita, P . Slavich, JHEP 1108, 128 (2011). arXiv:1107.0914

56. G. Degrassi, S. Di Vita, P. Slavich, Eur. Phys. J. C 72, 2032 (2012). arXiv:1204.1016
57. R. Harlander, M. Krämer, M. Schumacher, arXiv:1112.3478

58. E. Bagnaschi, R. Harlander, S. Liebler, H. Mantler, P. Slavich et al., JHEP 1406, 167 (2014). arXiv: 1404.0327

59. S. Heinemeyer, O. Stal, G. Weiglein, Phys. Lett. B 710, 201-206 (2012). arXiv:1112.3026

60. ATLAS collaboration, ATLAS-CONF-2013-090, ATLAS-COMCONF-2013-107

61. ATLAS collaboration, G. Aad et. al., JHEP 1503, 088 (2015). arXiv: 1412.6663

62. CMS Collaboration, CMS-PAS-HIG-14-020

63. T. Stefaniak, Higgs Couplings and Supersymmetry in the Light of early LHC Results. PhD thesis, Bonn University, 2014. http://hss. ulb.uni-bonn.de/2014/3697/3697.htm

64. J.F. Gunion, H.E. Haber, Phys. Rev. D 67, 075019 (2003). arXiv:hep-ph/0207010

65. N. Craig, J. Galloway, S. Thomas, arXiv:1305.2424

66. D. Asner, T. Barklow, C. Calancha, K. Fujii, N. Graf, et. al., arXiv:1310.0763. (See Chapter 1.3)

67. M. Carena, I. Low, N.R. Shah, C.E. Wagner, JHEP 1404, 015 (2014). arXiv:1310.2248

68. H. E. Haber, arXiv: 1401.0152

69. M. Carena, H.E. Haber, I. Low, N.R. Shah, C.E.M. Wagner, arXiv: 1410.4969

70. ATLAS collaboration, G. Aad et. al., arXiv:1408.7084

71. ATLAS collaboration, G. Aad et. al., arXiv:1408.5191

72. ATLAS collaboration, G. Aad et. al., arXiv:1412.2641

73. ATLAS collaboration, G. Aad et. al., JHEP1504, 117 (2015). arXiv: 1501.0494

74. ATLAS collaboration, G. Aad et. al., arXiv:1409.6212

75. CMS collaboration, V. Khachatryanet. al., Eur. Phys. J. C 74(10), 3076 (2014). arXiv: 1407.0558

76. CMS collaboration, S. Chatrchyan et. al., Phys. Rev. D 89, 092007 (2014). arXiv:1312.5353

77. CMS collaboration, S. Chatrchyan et. al., JHEP 1401, 096 (2014). arXiv: 1312.1129

78. CMS collaboration, S. Chatrchyan et. al., Nat. Phys. 10 (2014). arXiv: 1401.6527

79. CDF collaboration, T. Aaltonen et. al., Phys. Rev. D 88(5), 052013 (2013). arXiv:1301.6668

80. D0 collaboration, V. M. Abazov et. al., Phys. Rev. D 88(5), 052011 (2013). arXiv:1303.0823

81. O. Stål, T. Stefaniak, PoS EPS-HEP2013, 314 (2013). arXiv: 1310.4039

82. N. Blinov, D. E. Morrissey, JHEP 1403, 106 (2014). arXiv: 1310.4174

83. D. Chowdhury, R.M. Godbole, K.A. Mohan, S.K. Vempati, JHEP 02, 110 (2014). arXiv: 1310.1932 